\title{
Design and Implementation of an Ultra-Low-Power ECG Patch and Smart Cloud-Based Platform
}

This paper was downloaded from TechRxiv (https://www.techrxiv.org).

\section{LICENSE}

CC BY-NC-SA 4.0

SUBMISSION DATE / POSTED DATE

$12-11-2021 / 17-11-2021$

\section{CITATION}

Baraeinejad, Bardia (2021): Design and Implementation of an Ultra-Low-Power ECG Patch and Smart CloudBased Platform. TechRxiv. Preprint. https://doi.org/10.36227/techrxiv.17003401.v1

DOI

10.36227/techrxiv.17003401.v1 


\title{
Design and Implementation of an Ultra-Low-Power ECG Patch and Smart Cloud-Based Platform
}

\author{
Bardia Baraeinejad, Masood Fallah Shayan, Amir Reza Vazifeh, Diba Rashidi, Mohammad Saberi \\ Hamedani, Hamed Tavolinejad, Pouya Gorji, Parsa Razmara, Kiarash Vaziri, Daryoosh Vashaee, \\ Mohammad Fakharzadeh, Senior Member, IEEE
}

\begin{abstract}
This paper reports a new device for electrocardiogram (ECG) signal monitoring and software for signal analysis and artificial intelligence (AI) assisted diagnosis.

The hardware mitigates the signal loss common in previous products by enhancing the ergonomy, flexibility, and battery life. The power efficiency is optimized by design using switching converters, ultra-low-power components, and efficient signal processing. It enables 14-day of uninterrupted ECG monitoring and connectivity with a smartphone and microSD card storage.

The software is implemented in Android app and web-based platforms via Internet of Things (IoT). This component provides cloud-based and local storage and uses AI for arrhythmia detection. The arrhythmia detection algorithm shows $\mathbf{9 8 . 7 \%}$ accuracy using Artificial Neural Network and K-Nearest Neighbors methods, and $\mathbf{9 8 . 1 \%}$ using Decision Tree method on test data set.
\end{abstract}

Keywords - Arrhythmia Detection, Artificial Intelligence, Cardiovascular Diseases, Cloud Storage, Electrocardiogram (ECG), Internet of Things (IoT), Wearable Sensors

\section{INTRODUCTION}

$\mathrm{C}$ ardiovascular diseases (CVD) are the leading cause of death, estimated in 2019 to cause 18.6 million deaths per year worldwide [1], while $90 \%$ of such diseases are preventable [2]. Among them, cardiac arrhythmia is a common condition. Cardiac arrhythmia and fast heart beatings may lead to symptoms such as dizziness, pounding, shortness of breath, and forceful extra heartbeats [3]. These symptoms are often accompanied by chest pain or discomfort, diaphoresis, neck fullness, or vasovagal type of response with syncope, diaphoresis, or nausea in case of tachycardia [3]. On the other hand, all types of arrhythmia may be asymptomatic [3]. Asymptomatic cardiac arrhythmia may happen so sporadically that only through extensive ECG monitoring can it be detected [4]. The rarity of such cases should not lead to underestimating their importance, as they can lead to syncope, cardiac arrest, or sudden death. However, accessibility is a major issue regarding CVD monitoring - not every artifact is expected to occur continuously or during the clinical setting of recording ECG signals [5]. Moreover, clinical ECG devices utilize several electrodes running through multiple cables, which can be challenging to set correctly by non-clinicians outside the health care facilities. On the other hand, limited Holter monitoring, four days and below, is proven to be insufficient in detecting sporadic arrhythmias. Due to limited battery life, the current Holter monitor devices do not allow extended monitoring periods with high resolution (Table II). Recent research has proven the efficacy of monitoring through single-lead ECG patches as an alternative to Holter monitoring. In a clinical study, 7day monitoring with a single-lead ECG patch yielded more accurate results than typical 24-hour Holter monitoring [6].
In cases such as cerebral ischemic events, a minimum of 7day monitoring is required to detect atrial fibrillation [7], which could be lifesaving.

In 2020 and 2021, due to the health concerns regarding the COVID-19 pandemic, visiting clinics and hospitals for extended periods partially lost its feasibility, calling for alternative methods of monitoring ECG accurately at home and using telemetry for patient-doctor communication. Furthermore, ECG is proven to be a helpful measure for cardiovascular involvement in COVID-19 patients [8]. However, the utility of such devices is often overshadowed by existing hardware requiring the involvement of an independent analysis facility. While efforts to create online and smartphone-based platforms have been made, they are either rudimentary and outdated or lack the required detection capabilities compared to facility-based detection processes [9, Table II]. This article, details our endeavor to manufacture a device and software that allows non-stop monitoring of ECG signals via the Internet and the arrhythmia detection algorithm assisting in diagnosis.

This paper is organized as follows. Section I presents the design and manufacturing of a power-efficient, ergonomic, portable, single-lead ECG monitoring patch, connectable to a smartphone via Bluetooth Low Energy, and accommodating local storage for a microSD card. Section II presents the software, detailing DSP-based noise filtering, AI-assisted arrhythmia detection, patient profiling, the web service, and smartphone application that enables device-phone connection, real-time signal viewing, data collection, and storage.

\section{HARDWARE DESIGN}

\section{A. Hardware Overview}

Our objective is to introduce a lightweight, user-friendly, ultra-low-power alternative that can collect desired data, process it, store it, and send it to an Android device via a transition protocol, which can yield overall better results in terms of accuracy, resolution, sample rate and battery life than the current commercial devices.

\section{B. Block Diagram and Explanation of Each Part}

As shown in Fig. 1, the block diagram of the device includes an ARM Cortex M4F processor and multiple peripherals, including Bluetooth Low Energy 5 [10]. Moreover, the diagram shows a USB type C connected to a LiPo battery charger (for battery charging) [11], a microSD card connector (for the insertion of external memory and further data storage), a level translator (for communication between microSD card connector and MCU) [12], two pushbuttons (to turn the device on/off and to start Bluetooth advertising), a single-lead ECG Analog Front-End (which uses two electrodes and amplifies the differential voltage also known as ECG AFE) [13], an accelerometer (for motion 
detection of the user) [14], three LEDs and a buzzer (to indicate notifications), and two DC-DC step-down converters (which supply 1.8 Volt and 3.3 Volt power from the battery to all parts) [15].

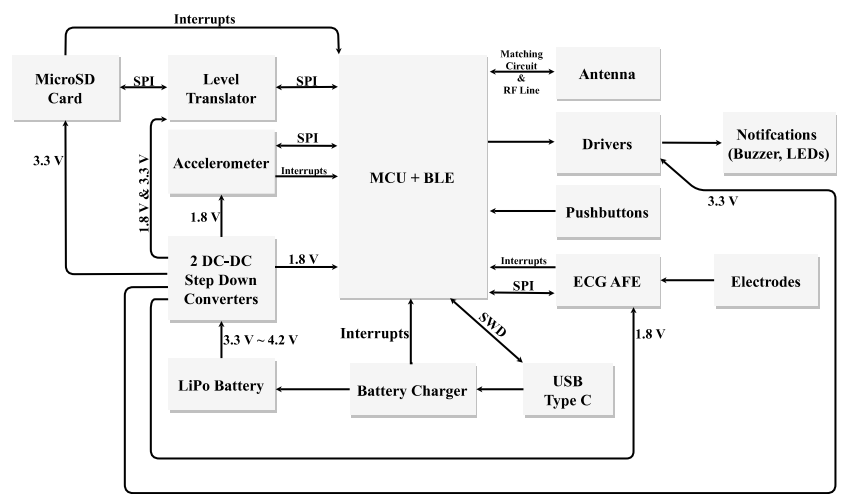

Fig. 1. Block diagram of the ECG patch hardware - The sensors, the BLE unit and the microcontroller run on $1.8 \mathrm{~V}$ except for microSD and the drivers connected to the interface elements running on 3.3V. Two step-down converters directly connect to the $3.3 \sim 4.2 \mathrm{~V}$ LiPo battery to power the mentioned sectors.

\section{1) Sensors - ECG AFE, Accelerometer}

Two sensors are integrated into the device: a single-lead ECG analog front-end (AFE) and an accelerometer. ECG AFE provides an electrocardiographic waveform through two electrodes connected via coaxial cables (shielded for noise reduction) to IPX connectors. A lead-on/lead-off detection feature allows the microcontroller to detect sensor-body attachment to lessen power consumption. The amplifier voltage gain within the sensor is programmable, ranging from $20 \mathrm{~V} / \mathrm{V}$ to $160 \mathrm{~V} / \mathrm{V}$. The $\mathrm{ADC}$ resolution is $18 \mathrm{bits}$ (the effective number of bits is 15.1 so we use 16 most significant bits, and the peak-to-peak noise is $5.44 \mu \mathrm{V}$ in the present device configuration). The power consumption of the ECG $\mathrm{AFE}$ is $180 \mu \mathrm{W}$ by measurement [13].

A 3-axis accelerometer is included to detect motion, track physical activity, and detect slips and falls. Furthermore, the data from the accelerometer enables detecting the noise caused by muscle artifacts and errors due to shakes. The sensor power consumption is $20 \mu \mathrm{W}$, per several measurements [14].

\section{2) Power Management}

A rechargeable $500 \mathrm{mAh}$ lithium-ion polymer battery is used to power the circuit. Two step-down DC-DC buck converters regulate the voltage to $3.3 \mathrm{~V}$ and $1.8 \mathrm{~V}$. These converters are highly efficient to assist in achieving a high battery life [15]. In addition, an integrated linear battery charger supplied by a USB type C port is used to recharge the battery [11]. A complete cycle takes around three hours. The choice of $500 \mathrm{mAh}$ battery capacity was made according to the overall current consumption of the device, i.e., $1.4 \mathrm{~mA}$ at $3.7 \mathrm{~V}$ battery voltage, enabling more than two weeks of battery life.

\section{3) Microcontroller and Peripherals}

The processing core is powered by a 32-bit ARM Cortex M4F running at $64 \mathrm{MHz}$. This processor was chosen due to its low power usage and hardware capabilities. The microcontroller is equipped with $32 \mathrm{KBs}$ of RAM and a 512 KB flash memory. SPI (Serial Peripheral Interface) is used for data transfer, with an instance for data from the sensors and another for microSD output. Serial Wire Debug (SWD) protocol was used to program this microcontroller. Two of the extra pins in the USB type C port were used for this process, one for SWDCLK and the other for SWDIO. A BLE 5 (Bluetooth Low Energy) transceiver sends data packets to and receives configurations from the connected Android device [10].

\section{4) Storage}

A microSD card can be optionally inserted into the device to save a backup of the data collected from the sensors in a format designed to be compatible with the web application (Refer to the section on web application for further explanation). Furthermore, a level translator supplies the $3.3 \mathrm{~V}$ needed for the microSD as opposed to the $1.8 \mathrm{~V}$ the microcontroller runs on [12].

\section{5) LEDs, Buzzer, and Pushbuttons}

Three LEDs are placed on the side of the device body, configured in three modes: alternating mode indicating BLE advertising, blinking mode indicating that the device is turned on and is functional, and a charging mode indicating the amount of battery charge as well as a buzzer to provide audible notifications of BLE being disconnected or a misplaced microSD. In addition, two pushbuttons are placed alongside the LEDs, one to exclusively enable Bluetooth advertising for one minute (marked with a Bluetooth logo), another for turning the device as well as Bluetooth advertising on normal press and turning the device off on long press (marked with a power on/off symbol).

\section{Printed Circuit Board Design}

The Printed Circuit Board (PCB), which is $0.8 \mathrm{~mm}$ thick, was designed to accommodate high-frequency parts and the industrial standards of body design. An electroless nickel immersion gold (ENIG) surface finish was chosen for the final manufactured board to ensure RoHS compliance (Fig. 2).
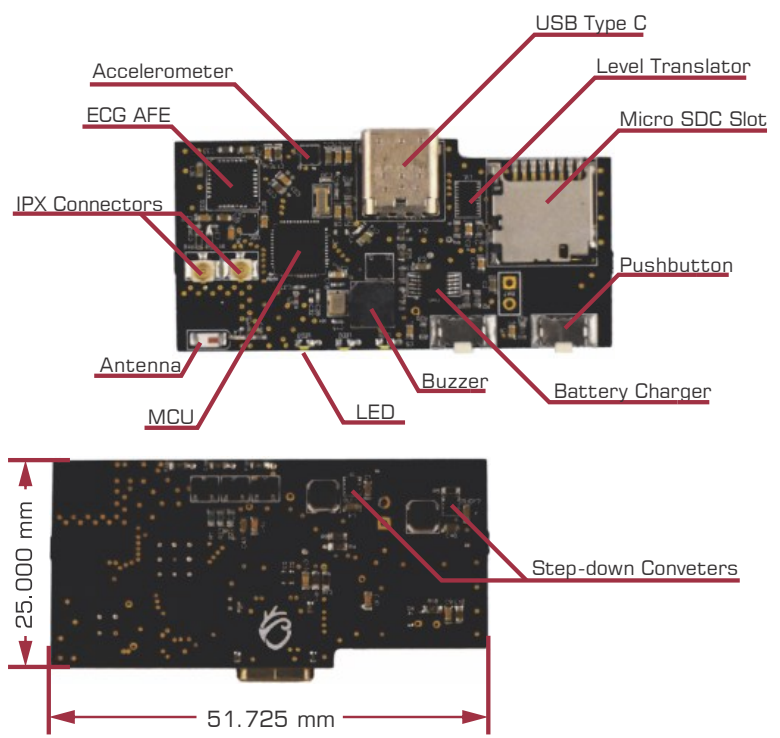

Fig. 2. Pictures from PCB top layout (top) and bottom layout (bottom). The critical parts and the PCB dimensions are marked.

\section{Hardware Code Flowchart}

The data collection timer affects the ECG data, recorded at 256 samples per second, and the accelerometer data, recorded at 50 samples per second. Every $50 \mathrm{~ms}$, a check runs as to whether a central BLE and/or a microSD is connected, and data from FIFO storage on the sensors is sent accordingly. 
Note that only FAT32 is currently supported for microSD storage. During the time no sensor interactions occur, the device goes into sleep mode.

The ADC timer measures battery every $500 \mathrm{~ms}$. A resistor divider scales the battery voltage to the microcontroller voltage to send a percentage level and charging status via BLE. Also, an individual UI timer orchestrates the LEDs and the buzzer.

As discussed in the previous section, the Bluetooth pushbutton calls an interrupt to initialize Bluetooth advertising and the power button to power the device on/off. Each sensor is individually configurable via BLE through the peripheral. The discussed procedure is shown in Fig. 3.

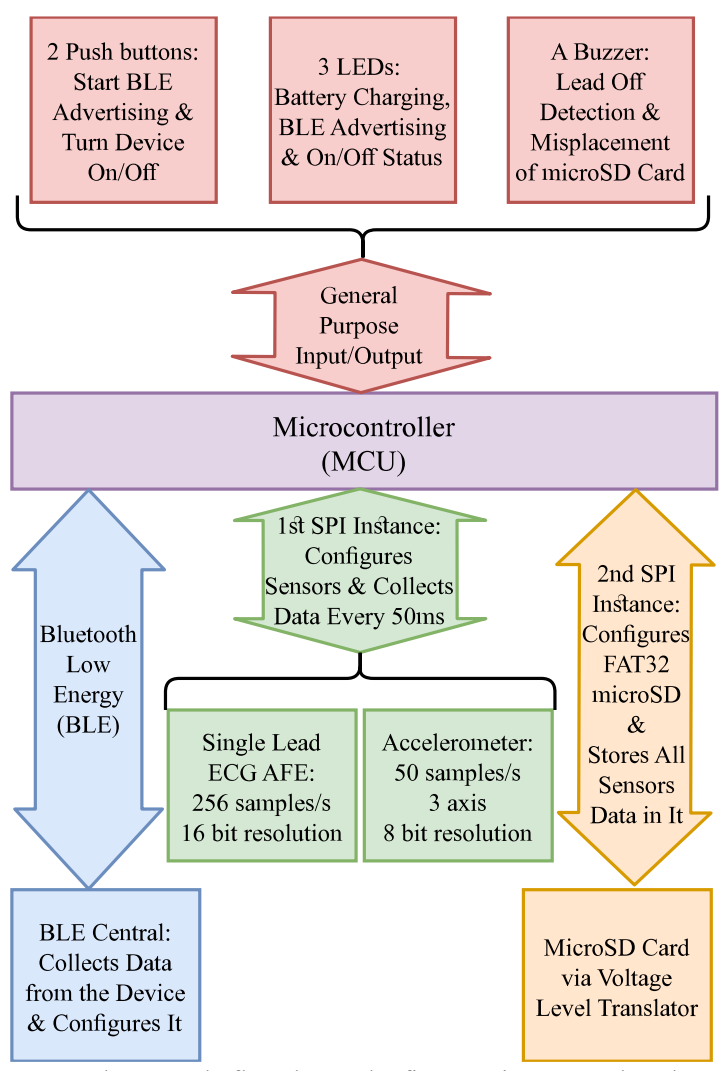

Fig. 3. Hardware code flowchart. The first SPI instance takes data from the accelerometer, the second takes microSD, and the rest of the hardware code functionality concerns interface elements from MCU input/output pins and device configuration via BLE.

\section{E. Body Design}

The body frame is 3D printed using HP Multi Jet Fusion technology, with PA12 medical grade material [16] featuring a curved ergonomic design. The intended setup is setting the device upright, which puts both electrodes on a lead near the lead II (Fig. 4). Lead II was chosen since it is more suitable for rhythm detection and gives a good view of the $\mathrm{P}$ wave [17]. The wings are curved in all three dimensions in two opposite directions aiming to allow unobstructed patient movement and improved signal accuracy and stability. The honeycomb design was adopted to provide more flexibility while improving the aesthetics. The outer curve of the wing conceals and protects the wiring connecting the patch to the circuit. The stainless-steel lid in the closed state covers the micro-SD slot and the USB Type-C jack. Furthermore, it increases the physical durability of the device. The medical practitioner can slide the lid upwards in its set constraints to access the slot and the jack.
The HP PA12 biocompatible material has a dense and strong structure with a balanced property profile [16]. The following certifications were considered in choosing this material: USP Class I-VI, US FDA guidance for Intact Skin Surface Devices, RoHS, EU REACH, and PAHs [16]. The side view, exploded view, critical parts and dimensions of the product are demonstrated in Fig. 5, Fig. 6, Fig. 7, and Fig. 8 respectively.
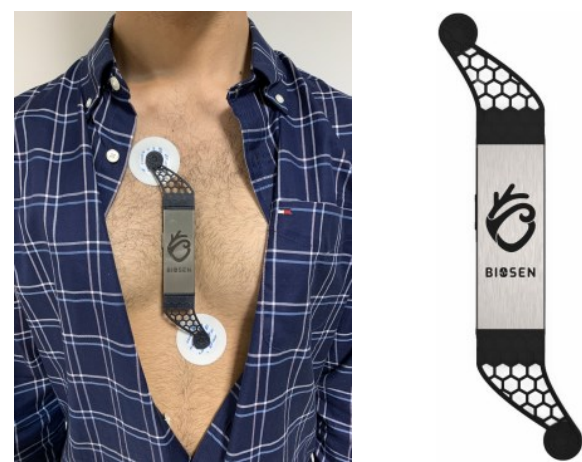

Fig. 4. The device on the body (left) and a render of the device (right)

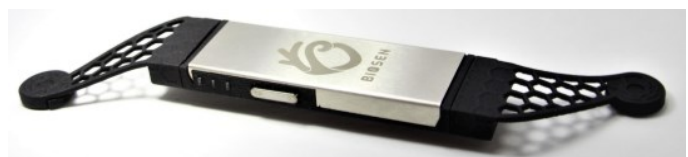

Fig. 5. A photograph of the final product

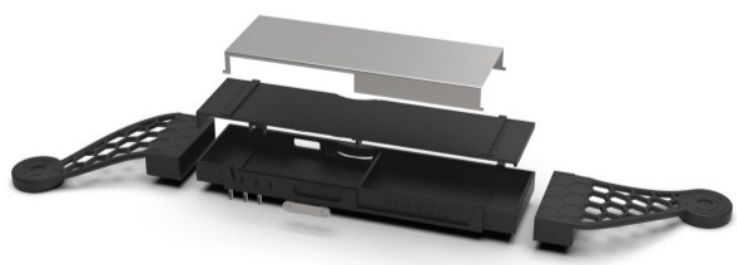

Fig. 6. A render of the exploded view of the body

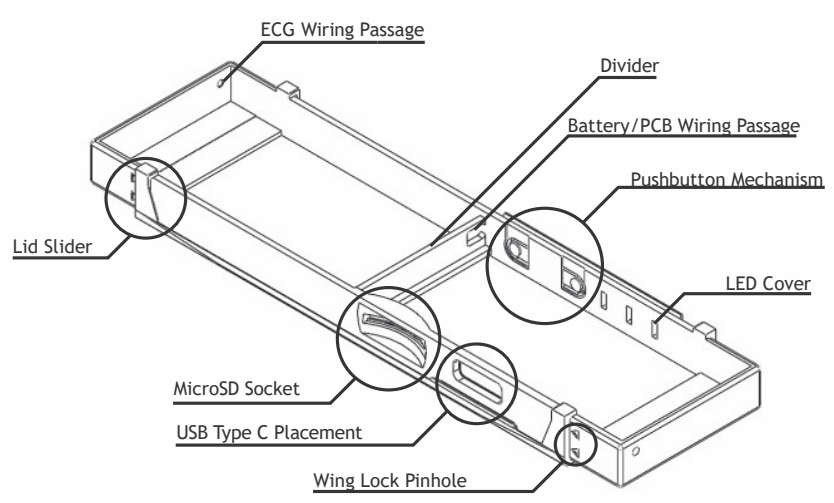

Fig. 7. Critical parts of the main enclosure
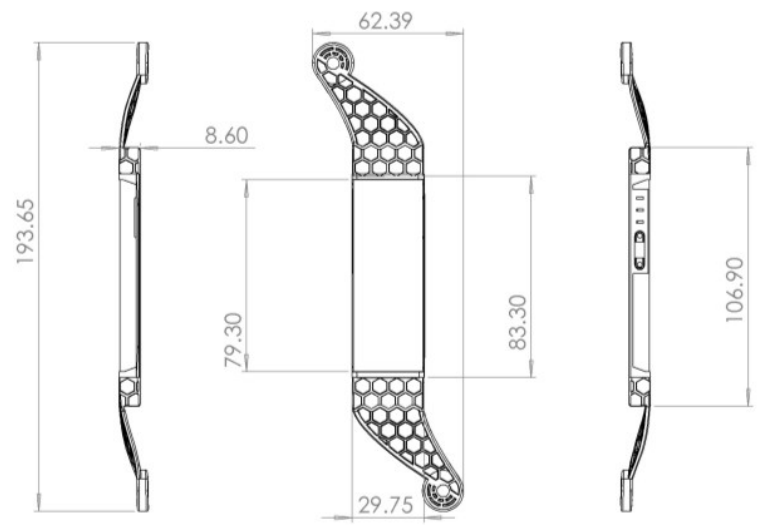

Fig. 8. Body dimensions (all dimensions in millimeters) 


\section{SOFTWARE DESIGN}

\section{A. Software Overview}

The software introduces a comprehensive platform for ECG signal viewing and processing, implemented as an Android application and a web application via Internet of Things (IoT). Server-side processing detects features such as R-to-R intervals and arrhythmias via artificial intelligence, and the Android application serves as a real-time signal viewer.

\section{B. Android Application and Web Application}

The following section covers the parts concerning the endusers and medical practitioners using the service.

\section{1) Web Application}

A practitioner can create and modify patient profiles through a web application accessible through a web browser. In addition, cloud storage allows complete access to patient data in and outside the office on multiple devices.

\section{2) Signal Analyzer Interface}

The microSD card data, alongside patient names, and sampling dates are saved on the cloud to enable viewing and analyzing ECG data remotely. Arrhythmia detection algorithms are triggered server-side, and then the output is shown on a webpage, visually marking arrhythmia and R-to$\mathrm{R}$ intervals in milliseconds. The user can edit generated annotations, add comments on any selected signal segment and measure the interval between any two selected points in seconds. The result can be exported to a PDF file (Fig. 9).

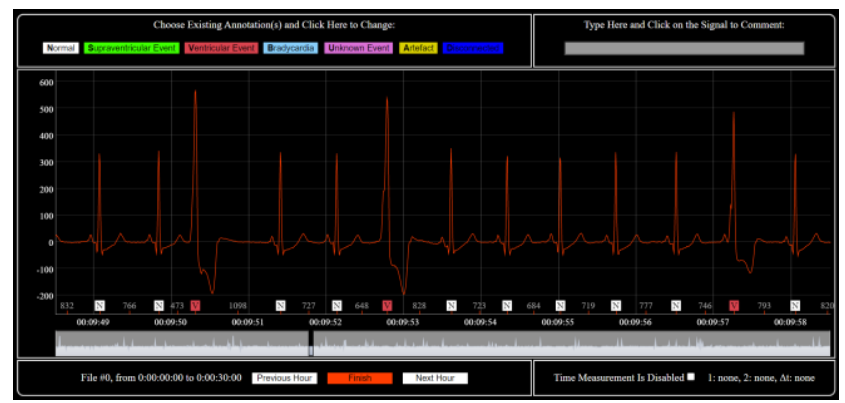

Fig. 9. A screenshot of the web application, demonstrating the analyzer interface.

\section{3) Android Application}

After logging in, the data received through BLE is displayed in real-time and can be partially stored locally. An option to configure external devices also exists (Fig. 10).
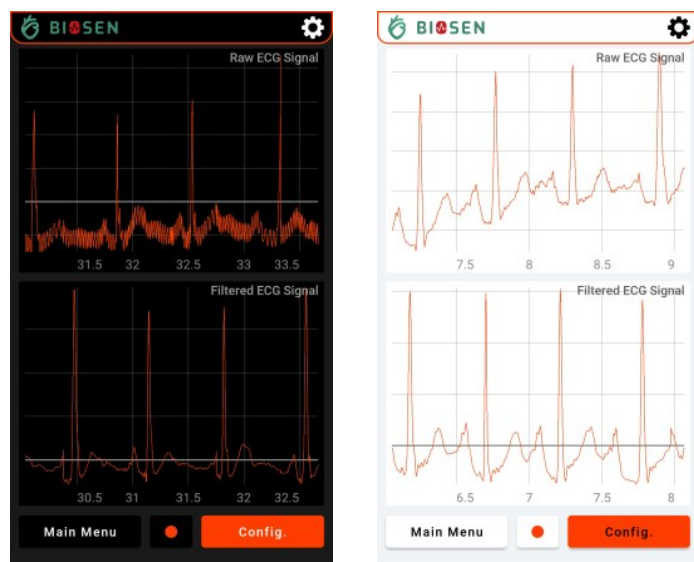

Fig. 10. A screenshot of the Android application demonstrating the signa before and after filtering in dark (left) and light (right) themes.

\section{4) Request Handling}

All HTTP requests sent from the web application and the android application (e.g., login forms) are received and processed through RESTful web service and stored on the server whenever needed. In addition, all patient and practitioner data are stored on a SQL-based database (MySQL), and recorded signal files are stored individually on the server, easily accessible by the uploader through the Internet (Fig. 11).

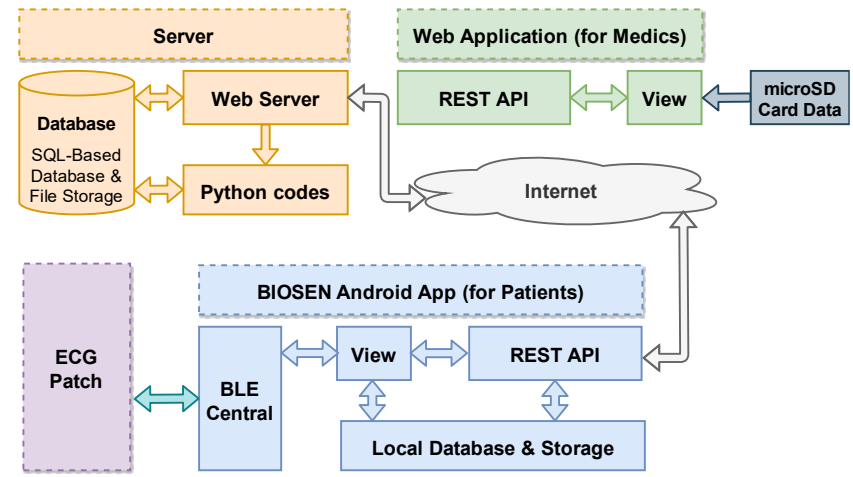

Fig. 11. The diagram of the web platform using IoT

\section{Server-Side Processing}

The following sector covers the server-side data analysis.

\section{1) Pre-Processing and Filtering}

The industrial standard for modern ECG recording is a bandpass ranging from $0.5 \mathrm{~Hz}$ to $100 \mathrm{~Hz}$ or $150 \mathrm{~Hz}$ [18]. Baseline wandering (BW) and Power Line Interference (PLI) are two significant noise sources in ECG signals. BW is a low-frequency artifact moving the $\mathrm{x}$-axis higher or lower than the intended fixed position. Patient movement, breathing, and improper electrodes can lead to $\mathrm{BW}$ noise. The frequency content range of $\mathrm{BW}$ noise is in the range of $0.5 \mathrm{~Hz}$. Another primary source of noise in ECG signals is power line interference (PLI). The frequency content range of PLI is either $50 \mathrm{~Hz}$ or $60 \mathrm{~Hz}$ (based on the location) with the appendage of specific harmonics [19]. The frequency sample rate of the ECG Patch is 256 samples per second, so its Nyquist frequency is equal to $128 \mathrm{~Hz}$, which covers the frequency content of the ECG signal. Therefore, based on the frequency range of ECG signals, it can be concluded that the desired filter should pass every frequency content in the range of $0.5 \mathrm{~Hz}$ up to $100 \mathrm{~Hz}$ except for $50 \mathrm{~Hz}$ (or $60 \mathrm{~Hz}$ ) in particular.

Various techniques like IIR notch filtering, FIR filtering, adaptive filtering, and filtered residue method have been proposed for ECG noise removal [20]. Unlike IIR filters consisting of impulse responses of infinite duration, FIR filters have finite impulse responses, which stabilizes the filter. FIR filters can be designed to have a linear phase and require no feedback. Also, designing an FIR filter tends to be a much more controlled process than IIR filters. The simplest method of FIR filter design is the windowing method, branching into different window types such as Hamming, Blackman, Rectangular, Kaiser, etc. [21]. Kaiser window provides a better ripple ratio and better main lobe width characteristic than other windows [22]. Therefore, as shown in Fig. 12, in the first stage of the filter, two FIR filters with the Kaiser Windowing method were utilized parallel to remove PLI.

Moreover, median filters are a common means in BW removal [23],[24]. Therefore, to increase the efficiency of 
BW removal, we used a median filter in the second stage. Thus, as shown in Fig. 13 and Fig. 14, the designed filter can remove PLI and BW simultaneously.

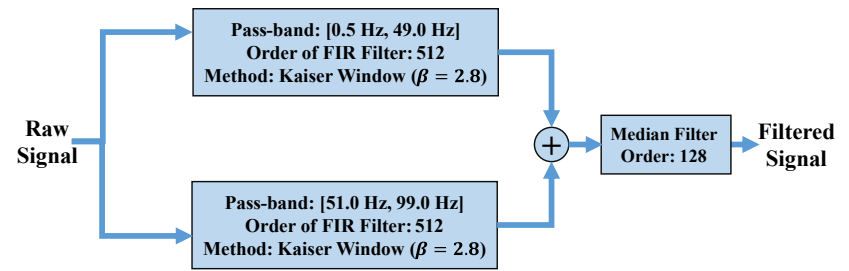

Fig. 12. Filter specifications - Initially, the signal is divided into two bands to discard the $50 \mathrm{~Hz}$ PLI noise and frequencies greater than $99 \mathrm{~Hz}$. Subsequently, a median filter is applied to remove the baseline wander.

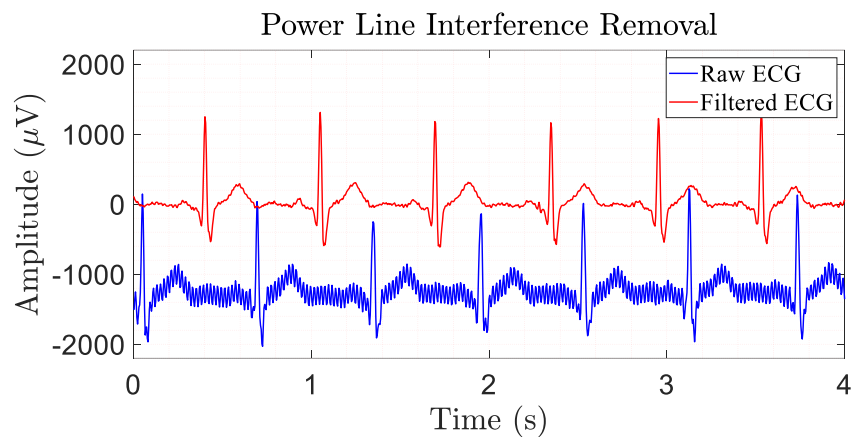

Fig. 13. PLI removal results using the proposed filter. The constant $50 \mathrm{~Hz}$ PLI noise is removed after the application of the filter.

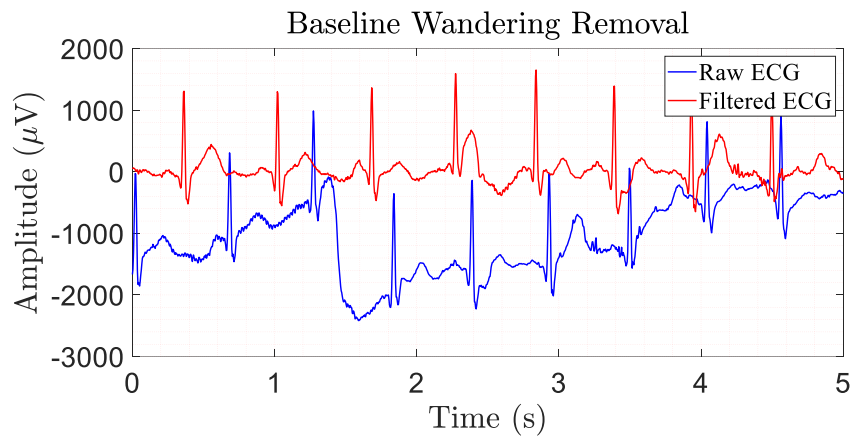

Fig. 14. BW removal results using the proposed filter. The resulting signal (red) has a fixed baseline after the application of the filter.

\section{2) R-peak Detection}

The ECG signal contains five significant peaks, known as Fiducial points, shown by letters P, Q, R, S, T [25], as seen in Fig. 15. Since the R-peak in the QRS complex is the most noticeable parameter for analyzing ECG signals, many algorithms have been proposed to detect R-peaks [26]. Some of these algorithms were examined and compared in terms of accuracy and runtime speed. The test results, shown in Table I, were obtained on our server. The accuracy level estimation is achieved through personal visual inspection. We have used the NeuroKit algorithm in our server since it results in higher accuracy in lower runtime.

\section{3) Dataset Analysis}

We use the MIT-BIH to provide an approach that can detect two significant types of arrhythmia. Unlike most prior works focused on only 2-class classification [33-38], we have examined three types of heartbeat annotation: normal, ventricular ectopic beat, and supraventricular ectopic beat. The labels 'A', 'S', 'a' and 'J' were used for supraventricular beat class, ' $\mathrm{V}$ ' and ' $\mathrm{E}$ ' for ventricular beat class, as well as ' $\mathrm{N}$ ', '.', 'L', 'R', 'e' and 'j' for normal class [38], [39]. Overall, there are 98476 heartbeats which include 88462 normal, 7235 ventricular beats, and 2779 supraventricular.

TABLE I

\begin{tabular}{ccc}
\hline \hline Algorithm & $\begin{array}{c}\text { Runtime on a 60 } \\
\text { minutes ECG } \\
\text { signal (s) }\end{array}$ & Accuracy \\
\hline Hamilton [27] & 1.44 & Low \\
Christov [28] & 15.39 & Low \\
Englese and Zeelenberg [29] & 6.22 & Low \\
Pan and Tompkins [30] & 6.24 & Medium \\
Stationary Wavelet Transform [31] & 0.75 & Medium \\
NeuroKit (slightly modified) [32] & 0.13 & High \\
\hline \hline
\end{tabular}

Comparison of different R-peak detection algorithms. Our modified version of the open-source NeuroKit algorithm significantly outperformed the other available methods in terms of runtime and accuracy.

\section{4) Feature Extraction}

Before feature extraction, a median filter shown in Fig. 12 is used for BW removal on each recording of the MIT-BIH dataset. We use the following segmentation method, which relies on R-peak detection, to separate every heartbeat signal, including all Fiducial points. As seen in Fig. 15, for each segment, the interval between the previous R-peak to the current is marked by $R_{n-1} R_{n}$. The last third of the $R_{n-1} R_{n}$ interval marks the beginning of each segment. The first twothirds of the $R_{n} R_{n+1}$ interval marks the end of the segment. We have extracted ten relatively quickly calculable features on each segment for different classes, which are variance of each segment to variance of corresponding recording ratio, skewness, kurtosis, RR interval, mean frequency, median frequency, OBW (frequencies occupying 99 percent of the bandwidth), band-power, previous RR interval to following $\mathrm{RR}$ interval ratio, and second derivative of RR interval. In addition to visual inspection with scattering features of different classes, shown in Fig. 16-a and Fig. 16-b, the Fisher score of each feature was calculated to separate effective features efficiently [40].

According to our findings on the MIT-BIH dataset, the following features tend to be more separative in classifying ventricular beats, supraventricular beats, and normal segments: second derivative of RR interval, previous RR interval to the following RR interval ratio, kurtosis, and Skewness.

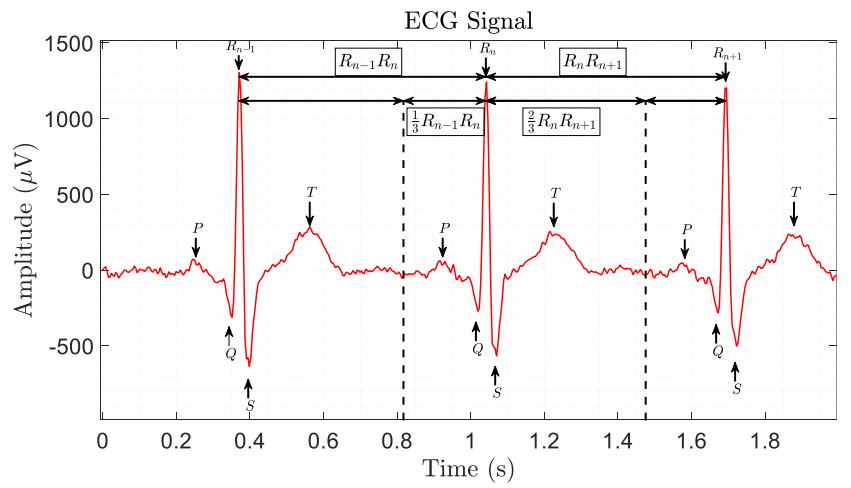

Fig. 15. A depiction of Fiducial points and segmentation selection.

\section{5) Pattern Classification}

After extracting features, artificial neural network (ANN), decision tree (DT), and K-nearest neighbor (KNN) were used for classification and compared in terms of accuracy and learning time. 

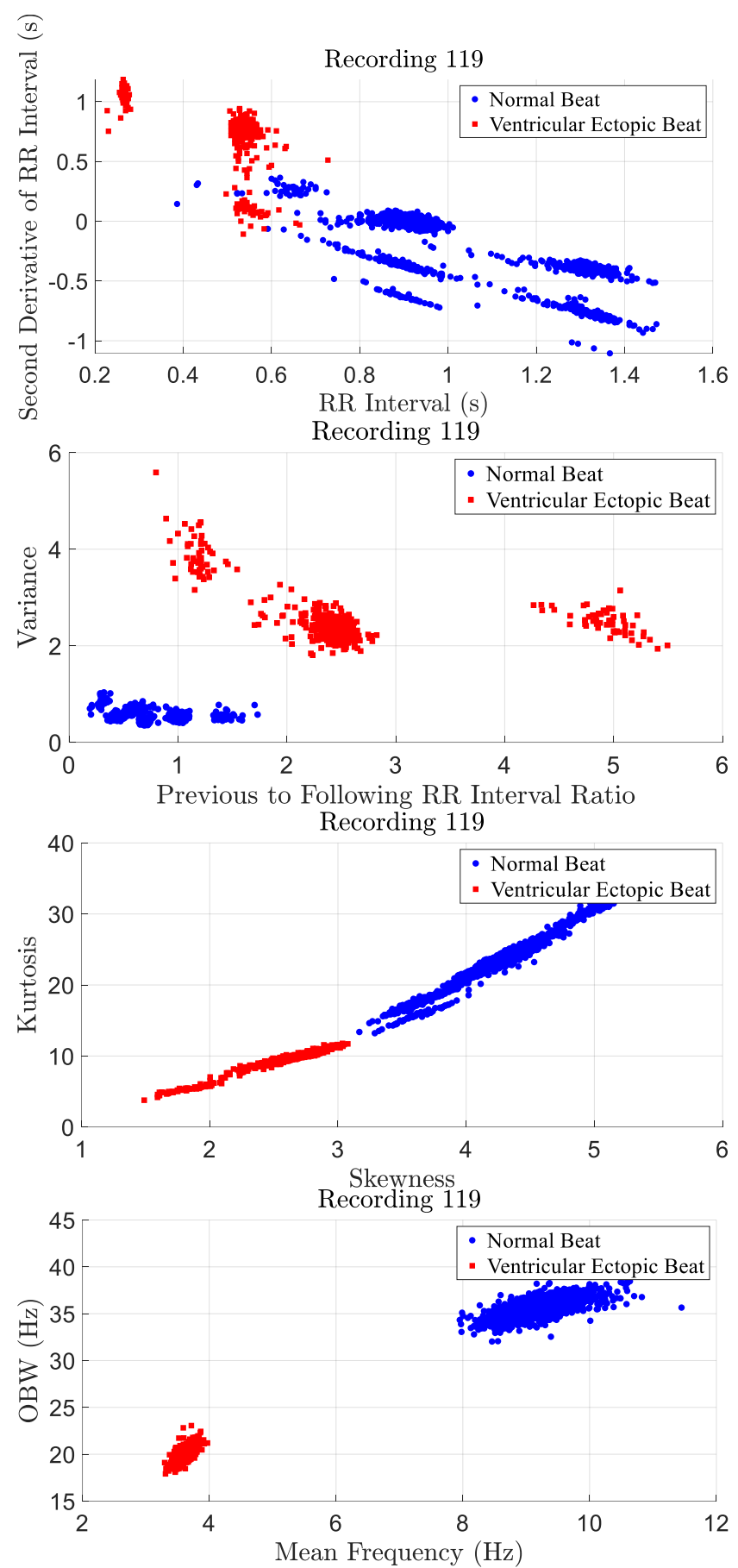

Fig. 16-a. The charts demonstrate the scattering of the features in recording 119 of MIT-BIH. As visible in the chart, the chosen criteria has a definitive separative quality.

\section{a) Artificial Neural Network (ANN)}

For ANN, we have used nprtool toolbox of MATLAB. The number of hidden neurons was set as 32 with 150 maximum number of iterations to avoid overfitting. All ten features were used in the process. Eighty percent of heartbeats were set as training data, and the other 20 percent were set as test data randomly. We tested three different training methods to reach higher accuracy, including Bayesian Regularization, Levenberg-Marquardt optimization, and scaled conjugate gradient. Based on our findings, Bayesian Regularization tends to have higher accuracy than other training algorithms. The confusion matrices of ANN with the Bayesian Regularization algorithm are shown in Fig. 17.
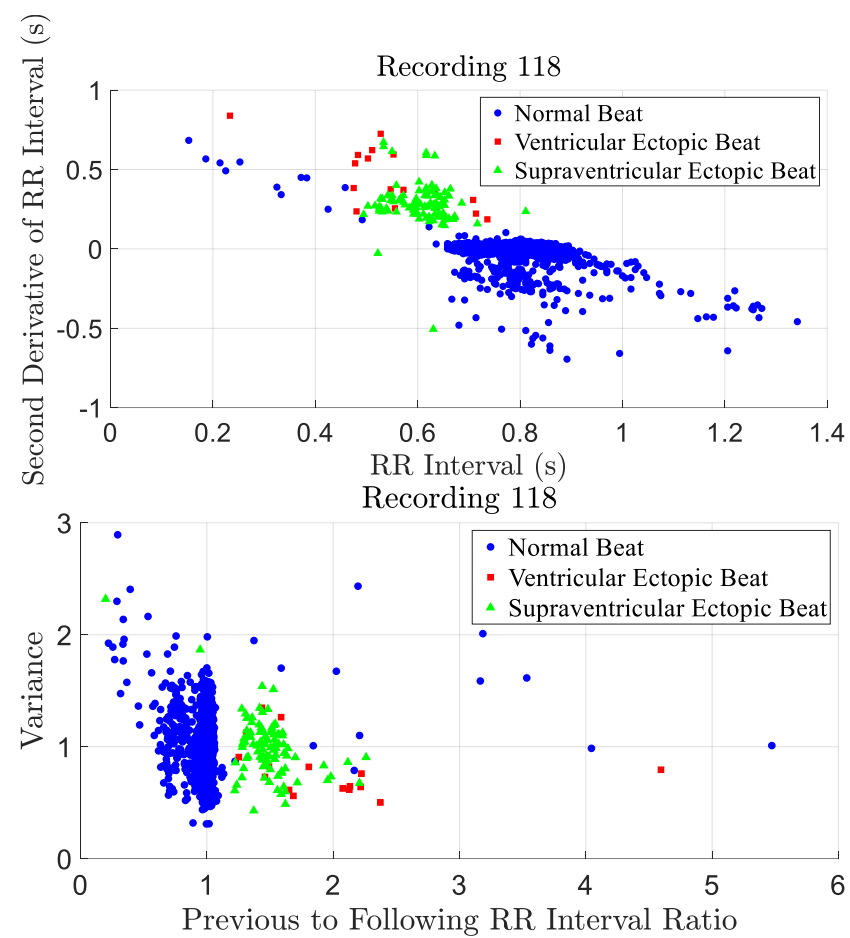

Fig. 16-b. The charts demonstrate the scattering of the features in recording 118 of MIT-BIH.

\section{b) Decision Tree (DT)}

The maximum number of splits is set as 1024 in the Decision Tree method to avoid overfitting, equal to 1.04 percent of total heartbeats samples. Identical training and test sets were used to provide a fair comparison between DT, $\mathrm{ANN}$, and KNN. Also, the same features were used in all classifiers. The confusion matrices of the Decision Tree are shown in Fig. 18.

\section{c) K-Nearest Neighbors (KNN)}

For KNN, we have used fitcknn function of MATLAB. The parameter $\mathrm{k}$, which decides how many neighbors will be chosen for $\mathrm{KNN}$ is set as 12. In addition, the distance function is set as seuclidean. The training set, test set, and features in the three classifiers are equal. The confusion matrices of the KNN are shown in Fig. 19.

In comparing the three classifiers, although Decision Tree seems to be the fastest and the most understandable classifier, ANN and KNN yield more accurate results in test data.

\section{6) Dataset Collection}

At the time of writing this article, this section of research is being evaluated for human subject research by National Committee for Ethics in Biomedical Researches (NCEBR) Institutional Review Board (IRB). ECG and accelerometer signals will be recorded and stored from patients and healthy candidates, who sign an agreement for anonymous inclusion of the recordings in a dataset. Random excerpts of recordings are to be evaluated and annotated by a medical doctor and uploaded on https://dataset.biosengroup.com for open-source usage. The findings of personal evaluation will be compared to the findings of our AI algorithm for debugging purposes. Upon further validation of the set, we plan to expand the training of our algorithm beyond the MIT-BIH set and improve its accuracy. 

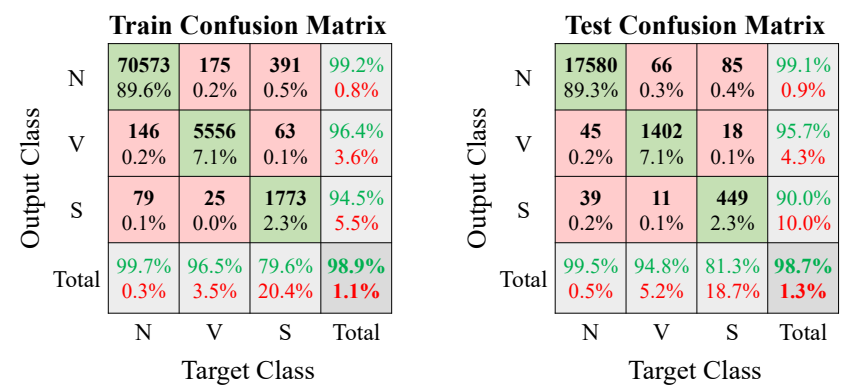

Fig. 17. ANN Train and Test Confusion Matrix ('S' stands for supraventricular ectopic beats, ' $\mathrm{V}$ ' for ventricular ectopic beats, and ' $\mathrm{N}$ ' for normal beats)
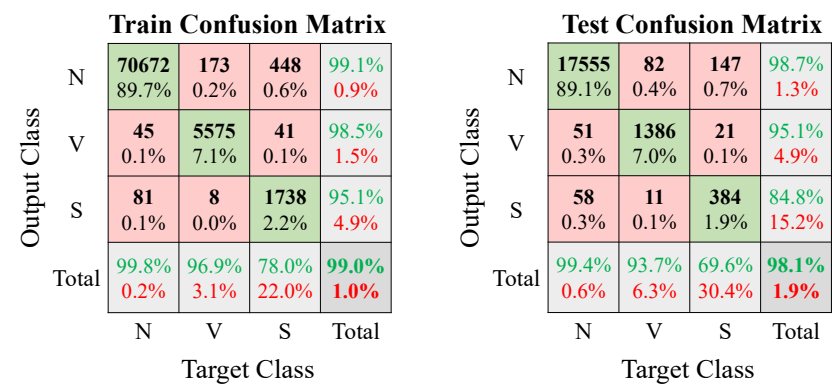

Fig. 18. DT Train and Test Confusion Matrix (' $S$ ' stands for supraventricular ectopic beats, ' $\mathrm{V}$ ' for ventricular ectopic beats, and ' $\mathrm{N}$ ' for normal beats)
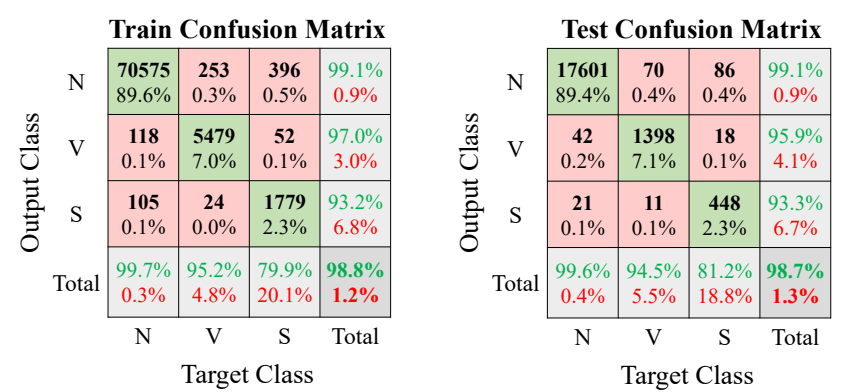

Fig. 19. KNN Train and Test Confusion Matrix (' $\mathrm{S}$ ' stands for supraventricular ectopic beats, ' $\mathrm{V}$ ' for ventricular ectopic beats, and ' $\mathrm{N}$ ' for normal beats)

\section{COMPARISON WITH EXISTING SYSTEMS}

This work was conceived as a research project to evolve into a commercial product. One of the primary factors was the commercial feasibility and offering enhanced connectivity and recording timeframe than the existing devices and software platforms. For this matter, we refrain from comparison with research concluded in a strictly academic environment with no actual clinical utility. As demonstrated in Table II, measures taken to extend the battery life appear to prolong the recording timeframe longer than the available clinically approved commercial alternatives. In addition, the connectivity methods and platform availability reduce the need for third-party involvement in the analysis process.

Furthermore, signal losses occurring due to artifacts, sudden muscle movements, and device-body disconnection appears to be rare due to the flexible structure.

The main challenge of the detection section was using a 3class system as opposed to the 2-class system commonplace in similar research while maintaining high accuracy. Table III demonstrates a comparison of various arrhythmia detection algorithms. Note that decreasing the server load required a decrease in the runtime of our algorithm. However, many similar researchers refrain from discussing runtime adequately to provide a comparison value.

\section{V.CONCLUSION}

The hardware developed in this project shows promise as an alternative to Holter monitoring and common ECG patch devices due to better battery performance and higher resolution and sample rate. In addition, longer recording timeframes allow the detection of more sporadic arrhythmias. The general hardware design needs little to no modification as of writing. Further research might focus on powering the device with a body energy harvester for prolonged or indefinite recording periods and the use of such recordings.

Cloud-based storage allows more accessible storage and management of data than similar products. The detection algorithm shows an improvement over existing research performed on the MIT-BIH dataset and could be improved upon by integrating the collected data set into the training set. Also, further research in the software component could be performed on the usage of accelerometer data to removal noise caused by motion artifacts.

\begin{tabular}{|c|c|c|c|c|}
\hline Device Name & $\begin{array}{l}\mathrm{ZIO} \\
\text { Patch }\end{array}$ & SEEQ MCT & $\begin{array}{c}\text { Generic } \\
\text { ECG Holter } \\
\text { Monitors }\end{array}$ & This Work \\
\hline $\begin{array}{l}\text { Data Storage } \\
\text { Timeframe }\end{array}$ & 14 days & 7.5 days & $24-72$ hours & $>14$ days \\
\hline $\begin{array}{c}\text { ECG Channel } \\
\text { Count }\end{array}$ & 1 & 1 & $\begin{array}{c}\text { Variable, } \\
\text { Ranging } \\
\text { from } 3 \text { to } 12\end{array}$ & 1 \\
\hline $\begin{array}{c}\text { ECG } \\
\text { Resolution } \\
\text { (bits) }\end{array}$ & 10 & 16 & Variable & 16 \\
\hline $\begin{array}{c}\text { ECG Sample } \\
\text { Rate }(\mathrm{Hz})\end{array}$ & 200 & 200 & Variable & 256 \\
\hline $\begin{array}{c}\text { Data } \\
\text { Transmission }\end{array}$ & $\begin{array}{l}\text { Return of } \\
\text { the } \\
\text { device } \\
\text { for data } \\
\text { retrieval }\end{array}$ & $\begin{array}{l}\text { Bluetooth } \\
\text { and cellular } \\
\text { transmission }\end{array}$ & $\begin{array}{l}\text { Local } \\
\text { download in } \\
\text { clinic }\end{array}$ & $\begin{array}{c}\text { BLE, } \\
\text { microSD } \\
\text { Card }\end{array}$ \\
\hline Weight (g) & 34 & 50 & $\begin{array}{c}\text { Variable } \\
\text { (more than } \\
100)\end{array}$ & 40 \\
\hline
\end{tabular}

A comparison of this work and commercial devices [41].

TABLE III

\begin{tabular}{|c|c|c|c|c|}
\hline Accuracy & Classifier(s) & Features & Type Detection & Ref. \\
\hline $92.25 \%$ & $\begin{array}{c}\text { KNN, DT, } \\
\text { Probabilistic } \\
\text { Neural } \\
\text { Network }\end{array}$ & 8 Features & $\begin{array}{l}\text { Premature } \\
\text { Ventricular } \\
\text { Contractions }\end{array}$ & {$[33]$} \\
\hline $98.90 \%$ & $\begin{array}{c}\text { Learning } \\
\text { Vector } \\
\text { Quantization } \\
\text { Neural } \\
\text { Network }\end{array}$ & $\begin{array}{l}\text { Lyapunov } \\
\text { Exponent } \\
\text { Curve }\end{array}$ & $\begin{array}{c}\text { Premature } \\
\text { Ventricular } \\
\text { Contractions }\end{array}$ & {$[34]$} \\
\hline $95.40 \%$ & $\begin{array}{l}\text { Multilayer } \\
\text { Perceptron }\end{array}$ & 11 features & $\begin{array}{c}\text { Premature } \\
\text { Ventricular } \\
\text { Contractions }\end{array}$ & {$[35]$} \\
\hline $99.70 \%$ & $\mathrm{KNN}$ & $\begin{array}{l}\text { Deep learning } \\
\text { model to } \\
\text { extract spatial } \\
\text { features }\end{array}$ & $\begin{array}{l}\text { Premature } \\
\text { Ventricular } \\
\text { Contractions }\end{array}$ & {$[36]$} \\
\hline $91.10 \%$ & Random forest & 16 features & $\begin{array}{c}\text { Supraventricular } \\
\text { Tachycardia }\end{array}$ & {$[37]$} \\
\hline $98.7 \%$ & $\begin{array}{l}\text { ANN, DT, } \\
\text { and KNN }\end{array}$ & 10 features & $\begin{array}{c}\text { Ventricular and } \\
\text { Supraventricular } \\
\text { beats }\end{array}$ & $\begin{array}{l}\text { This } \\
\text { Work }\end{array}$ \\
\hline
\end{tabular}

A comparison of this project and similarly arrhythmia detection papers. 


\section{REFERENCES}

[1] G. A. Roth et al. "Global Burden of Cardiovascular Diseases and Risk Factors, 1990-2019: Update from the GBD 2019 Study." Journal of the American College of Cardiology vol. 76,25 (2020): 2982-3021. doi: 10.1016/j.jacc.2020.11.010

[2] B.V. Reamy et al. "Prevention of Cardiovascular Disease." Primary care vol. 45,1 (2018): 25-44. doi: 10.1016/j.pop.2017.11.003

[3] D. Fu. "Cardiac Arrhythmias: Diagnosis, Symptoms, and Treatments." Cell biochemistry and biophysics vol. 73,2 (2015): 291-296. doi:10.1007/s12013-015-0626-4

[4] N. Dagres et al. ": Influence of the duration of Holter monitoring on the detection of arrhythmia recurrences after catheter ablation of atrial fibrillation: implications for patient follow-up." International journal of cardiology vol. 139,3 (2010): 305-6. doi: 10.1016/j.ijcard.2008.10.004

[5] J. J. Oresko et al. "A wearable smartphone-based platform for real-time cardiovascular disease detection via electrocardiogram processing." IEEE transactions on information technology in biomedicine: a publication of the IEEE Engineering in Medicine and Biology Society vol. 14,3 (2010): 73440. doi:10.1109/TITB.2010.2047865

[6] P. M. Barrett et al. "Comparison of 24-hour Holter monitoring with 14day novel adhesive patch electrocardiographic monitoring." The American journal of medicine vol. 127,1 (2014): 95.e11-7. doi: 10.1016/j.amjmed.2013.10.003

[7] R. Stahrenberg et al. "Enhanced detection of paroxysmal atrial fibrillation by early and prolonged continuous holter monitoring in patients with cerebral ischemia presenting in sinus rhythm." Stroke vol. 41,12 (2010): 2884-8. doi:10.1161/STROKEAHA.110.591958

[8] S. Haseeb et al. "Value of electrocardiography in coronavirus disease 2019 (COVID-19).” Journal of electrocardiology vol. 62 (2020): 39-45. doi: 10.1016/j.jelectrocard.2020.08.007

[9] A. Pantelopoulos and N. G. Bourbakis, "A Survey on Wearable SensorBased Systems for Health Monitoring and Prognosis," in IEEE Transactions on Systems, Man, and Cybernetics, Part C (Applications and Reviews), vol. 40, no. 1, pp. 1-12, Jan. 2010, doi: 10.1109/TSMCC.2009.2032660.

[10] "nRF52832 Product Specification v1.4", Nordic Semiconductor, Available: https://infocenter.nordicsemi.com/pdf/nRF52832_PS_v1.4.pdf

[11] "BQ2409x 1-A, Single-Input, Single-Cell Li-Ion and Li-Pol Battery Chargers", Texas Instruments, Available: https://www.ti.com/lit/gpn/bq24092

[12] TXB0108 8-Bit Bidirectional Voltage-Level Translator with AutoDirection Sensing and $\pm 15-\mathrm{kV}$ ESD Protection, Texas Instruments, Available: https://www.ti.com/lit/gpn/TXB0108

[13] "Ultra-Low Power, Single-Channel Integrated Biopotential (ECG, R-toR Detection) AFE (MAX30003)", Analog Devices (Maxim Integrated), https://datasheets.maximintegrated.com/en/ds/MAX30003.pdf

[14] "MEMS digital output motion sensor: ultra-low-power highperformance 3-axis "femto" accelerometer (LIS2DH12)", ST, Available: https://www.st.com/resource/en/datasheet/lis2dh12.pdf

[15] "TPS62840 1.8-V to 6.5-V, 750-mA, 60-nA IQ Step-Down Converter", Texas Instruments, Available: https://www.ti.com/lit/gpn/tps62840

[16] "HP 3D High Reusability PA 12 Datasheet", CimQuest, Accessed on 21/6/2021 [Online], Available: https://cimquest-inc.com/resourcecenter/HP/Materials/HP-PA12-Datasheet.pdf

[17] S. Meek, and F. Morris. "ABC of clinical electrocardiography.Introduction. I-Leads, rate, rhythm, and cardiac axis." BMJ (Clinical research ed.) vol. 324,7334 (2002): 415-8. doi:10.1136/bmj.324.7334.415

[18] L.G. Tereshchenko, and M. E. Josephson. "Frequency content and characteristics of ventricular conduction." Journal of electrocardiology vol. 48,6 (2015): 933-7. doi: 10.1016/j.jelectrocard.2015.08.034

[19] R. Kher, "Signal Processing Techniques for Removing Noise from ECG Signals." J Biomed Eng 1: 1-9., Accessed: 8/6/2021, Available: http://www.jscholaronline.org/articles/JBER/Signal-Processing.pdf

[20] A. Banerjee, M. H. Kolekar, L, Garg, B. Chakraborty, Chapter 6 of "Internet of Things for Healthcare Technologies", Springer-Verlag, New York; Berlin, Germany; Vienna, Austria

[21] A. V. Oppenheim and R. W. Schafer, Chapter 7 of "Discrete-time Signal Processing", $3^{\text {rd }}$ ed., Pearson; USA

[22] T. Kaya, M. C. Ince, "The Obtaining of Window Function Having Useful Spectral Parameters by Helping of Genetic Algorithm", Procedia Social and Behavioral Sciences, Vol. 83, 2013, Pg. 563-568, ISSN $1877-$ 0428, https://doi.org/10.1016/j.sbspro.2013.06.107.

[23] A. E. Awodeyi, S. R. Alty and M. Ghavami, "Median Filter Approach for Removal of Baseline Wander in Photoplethysmography Signals," 2013 European Modelling Symposium, 2013, pp. 261-264, doi: 10.1109/EMS.2013.45.

[24] Y. Xin et al. "ECG baseline wander correction based on mean-median filter and empirical mode decomposition." Bio-medical materials and engineering vol. 24,1 (2014): 365-71. doi:10.3233/BME-130820
[25] J. Aspuru et al. "Segmentation of the ECG Signal by Means of a Linear Regression Algorithm." Sensors (Basel, Switzerland) vol. 19,4 775. 14 Feb. 2019, doi:10.3390/s19040775

[26] X. Benlian, P. Jeong-Seon, L. Sang-Woong, P. Unsang. "R Peak Detection Method Using Wavelet Transform and Modified Shannon Energy Envelope". Journal of Healthcare Engineering https://doi.org/10.1155/2017/4901017

[27] P. Hamilton "Open-Source ECG Analysis", EP Limited, Somerville, USA, Accessed on 16/6/2021, Available: http://www.cinc.org/old/Proceedings/2002/pdf/101.pdf

[28] I.I. Christov, Real time electrocardiogram QRS detection using combined adaptive threshold. BioMed Eng OnLine 3, 28 (2004). https://doi.org/10.1186/1475-925X-3-28

[29] Implementation of W. Engelse and C. Zeelenberg, "A single scan algorithm for QRS detection and feature extraction", IEEE Comp. in Cardiology, vol. 6, pp. 37-42, 1979 with modifications A. Lourenco, H. Silva, P. Leite, R. Lourenco and A. Fred, "Real Time Electrocardiogram Segmentation for Finger Based ECG Biometrics", BIOSIGNALS 2012, pp. 49-54, 2012. Usage

[30] J. Pan, W. J. Tompkins," A Real-Time QRS Detection Algorithm", IEEE Transactions on Biomedical Engineering, Vol. BME-32, No. 3, 1985 Available:

https://www.robots.ox.ac.uk/ gari/teaching/cdt/A3/readings/ECG/Pan+To mpkins.pdf

[31] V. Kalidas and L. Tamil, "Real-time QRS detector using Stationary Wavelet Transform for Automated ECG Analysis," in 2017 IEEE 17th International Conference on Bioinformatics and Bioengineering (BIBE), Washington, DC, USA, 2017 pp. 457-461. doi: 10.1109/BIBE.2017.00-12

[32] D. Makowski, T. Pham, Z. J. Lau, J. C. Brammer, F. Lespinasse, H. Pham, C. Schölzel, S. A. Chen, (2021). NeuroKit2: A Python toolbox for neurophysiological signal processing. Behavior Research Methods. https://doi.org/10.3758/s13428-020-01516-y

[33] T.S. Hock et al. "Automated Detection of Premature Ventricular Contraction Using Recurrence Quantification Analysis on Heart Rate Signals",Journal of Medical Imaging and Health Informatics DOI:10.1166/jmihi.2013.1181

[34] X. Liu, H. Du, G. Wang, S. Zhou, H. Zhang,

"Automatic diagnosis of premature ventricular contraction based on Lyapunov exponents and LVQ neural network,Computer", Methods and Programs in Biomedicine, Volume 122, Issue 1, 2015, Pages 47-55, ISSN 0169-2607, https://doi.org/10.1016/j.cmpb.2015.06.010.

[35] A. Ebrahimzadeh, A. Khazaee, "Detection of premature ventricular contractions using MLP neural networks: A comparative study", Measurement, Volume 43, Issue 1, 2010, Pages 103-112, ISSN 0263-2241, https://doi.org/10.1016/j.measurement.2009.07.002.

[36] J. Yu et al. "Automatic Premature Ventricular Contraction Detection Using Deep Metric Learning and KNN.”Biosensors vol. 11,3 69. 4 Mar. 2021, doi:10.3390/bios11030069

[37] Z. Li et al., "Supraventricular Tachycardia Detection via Machine Learning Algorithms," 2018 IEEE International Conference on Bioinformatics and Biomedicine (BIBM), 2018, pp. 2419-2422, doi: 10.1109/BIBM.2018.8621164.

[38]: B. Rajoub, "Machine learning in biomedical signal processing with ECG applications", Chapter 4, Pages 91-112,

Developments in Biomedical Engineering and Bioelectronics, Biomedical Signal Processing and Artificial Intelligence in Healthcare, Academic Press, 2020, https://doi.org/10.1016/B978-0-12-818946-7.00004-4.

[39] G. B. Moody, "MIT-BIH Arrhythmia Database Directory", Hypertext edition, Harvard-MIT Division of Health Sciences and Technology Biomedical Engineering Center, 1997, Available: https://physionet.org/physiobank/database/html/mitdbdir/intro.htm

[40] Q. Gu \& Z. Li \& J. Han. (2012). Generalized Fisher Score for Feature Selection. Proceedings of the 27th Conference on Uncertainty in Artificial Intelligence, UAI 2011.

Available: https://arxiv.org/ftp/arxiv/papers/1202/1202.3725.pdf

[41] E. Fung et al. "Electrocardiographic patch devices and contemporary wireless cardiac monitoring." Frontiers in physiology vol. 6 149. 27 May. 2015, doi:10.3389/fphys.2015.00149 